This item was submitted to Loughborough's Institutional Repository (https://dspace.lboro.ac.uk/) by the author and is made available under the following Creative Commons Licence conditions.

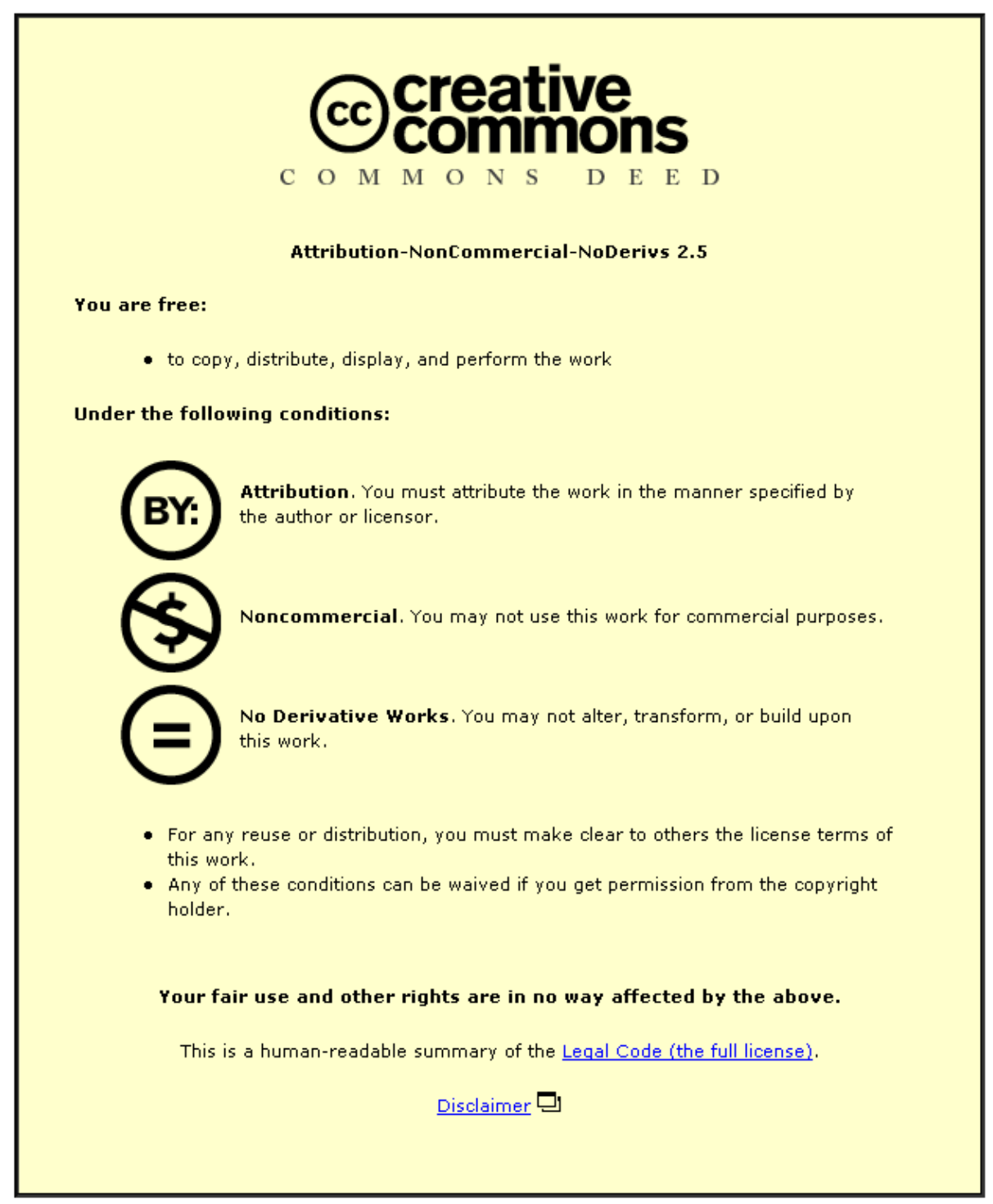

For the full text of this licence, please go to: http://creativecommons.org/licenses/by-nc-nd/2.5/ 


\title{
Eyes Extraction from Facial Images Using Edge Density
}

\author{
Muhammad Shafi and Paul W. H. Chung
}

\begin{abstract}
This paper proposes a novel method for eyes extraction in facial images using edge density information. The method is based on the observation that irrespective of skin colour, colour variations occur the most in the eye region. In the proposed method, edges are detected in the input facial image. Morphological dilation is applied twice and the holes are filled in the connected regions. This makes the high density edges regions appear as blobs. Certain shapes and geometrical rules are applied to these blobs to extract the eyes. The method was tested using images from the PICS facial images database. The accuracies of the initial blobs extraction and the final eyes extraction were $95 \%$ and $72 \%$ respectively.
\end{abstract}

\section{INTRODUCTION}

Eyes localization in facial images is very important in computer vision applications such as face recognition, face verification, gaze estimation and human computer interaction. The position of the eyes can be extracted from an image using different eye features such as colour, illumination, edge, shape and geometrical information. Most colour variations occur in eye regions in facial images due to colour difference between eyelids and skin, skin and sclera, sclera and iris, and iris and pupil (see figure 1). The colours of the rest of the face are more uniform when compared to the eyes. Therefore, if edge detection was applied to facial images then the eye regions would have maximum edge densities. The proposed method uses this edge density information to extract the eyes from facial images.

It is assumed in this paper that a facial image satisfies the following conditions.

- The input image is a head-shoulder image of a person such as passport photo.

- Both the eyes are visible (the direction of the head is within +-45 degrees).

- The face is not tilted more than 45 degrees towards the right or the left.

- The eyes are not closed.

The rest of the paper is organised as follow. Section 2 discusses the related work in the literature; section 3 gives details of the proposed method, section 4 discusses the experimental results when the proposed method is compared with two existing algorithms. Section 5 concludes the paper.

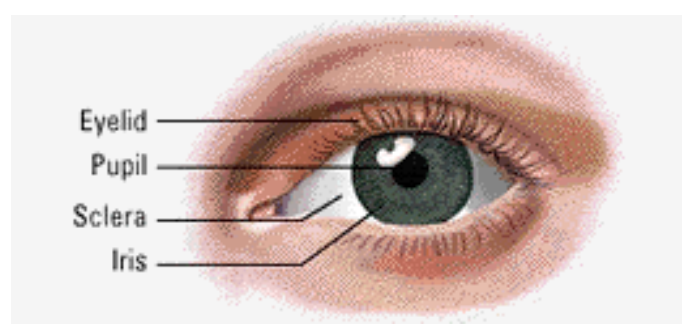

Figure 1 A Typical Eye Structure adopted from [7]

\section{RELATED WORK}

There has been extensive research in automatic eyes extraction for about two decades. Some of the face detection schemes also extract the eyes as the first step in face detection. Due to limited space it would not be possible to review all eye extraction methods, only a selected few are discussed here.

The red eye effect based eye detector is very robust and fast $[3,4]$. However, there are three issues with this proposed scheme: 1) need of specialized hardware, e.g. IBM PupilCam; 2) applicable to only videos; 3) the video needs to be taken at a short distance from the face in order to get the reflection from the pupils. Hsu, Mottaleb and Jain proposed an illumination-based eye detection method [1]. Although their method is quite robust, it is computationally expensive and it only works for colour images. Chiang, Tai, Yang, Huang and Huang proposed an eye detector based on the darkest part of the face [5]. In their method, the colour image is converted to equalized grey-level histogram A threshold is applied to identify the eyes. Although this method is quite fast, it fails to work with images of people with dark skin. The reason is that the eyes are not always the darkest parts of the face of people with very dark skin. Kawaguchi, Rizon and Hidaka proposed an eye detector that is based on Hough transform and separability filter [6]. The problem with this method is that it requires the rough size of pupil to be known. Also, 
due to circle fitting in Hough transform, it is computationally expensive.

\section{PROPOSED METHOD}

The propose method works on the basis that edge density is high around the eyes. After edge detection, morphological dilation and erosion are used to enhance the identified blobs and unnecessary small edges are removed. Shape and geometry information is used to extract and verify the eyes. The algorithm is given below then followed by a more detailed description of the important steps in the algorithm. The flow diagram is shown in figure 2 .

\section{ALGORITHM}

Input: head shoulder colour image

Output: head shoulders colour image with rectangles drawn around the two eyes

Start

Step A: Lighting Compensation

Convert the Coloured Image to Grey Level

Do histogram Equalization

Step B: Edge Detection

Detect Edges Using Sobel Operator

Step C: Dilation, Holes Filling and Erosion

Dilate the Image Twice Successively

Fill the Small Holes inside Connected Regions

Erode the Image Three Times Successively

Step D: Eyes Extraction and Verification

Apply the Shape and Geometry Based Rules and Reject the Regions those don't Satisfy These Rules

Draw Rectangles around the Remaining Connected Regions

End

\section{A. Lighting Compensation}

Some of the images in the PICS database have very poor contrast due to low lighting condition. If edge detection was applied directly to these images then the sharp edges are not identified properly. Therefore, the contrast of the images is adjusted first to get better results. Simple histogram equalization has been used in our experiments for contrast adjustment. Figure 3 shows the result of histogram equalization.

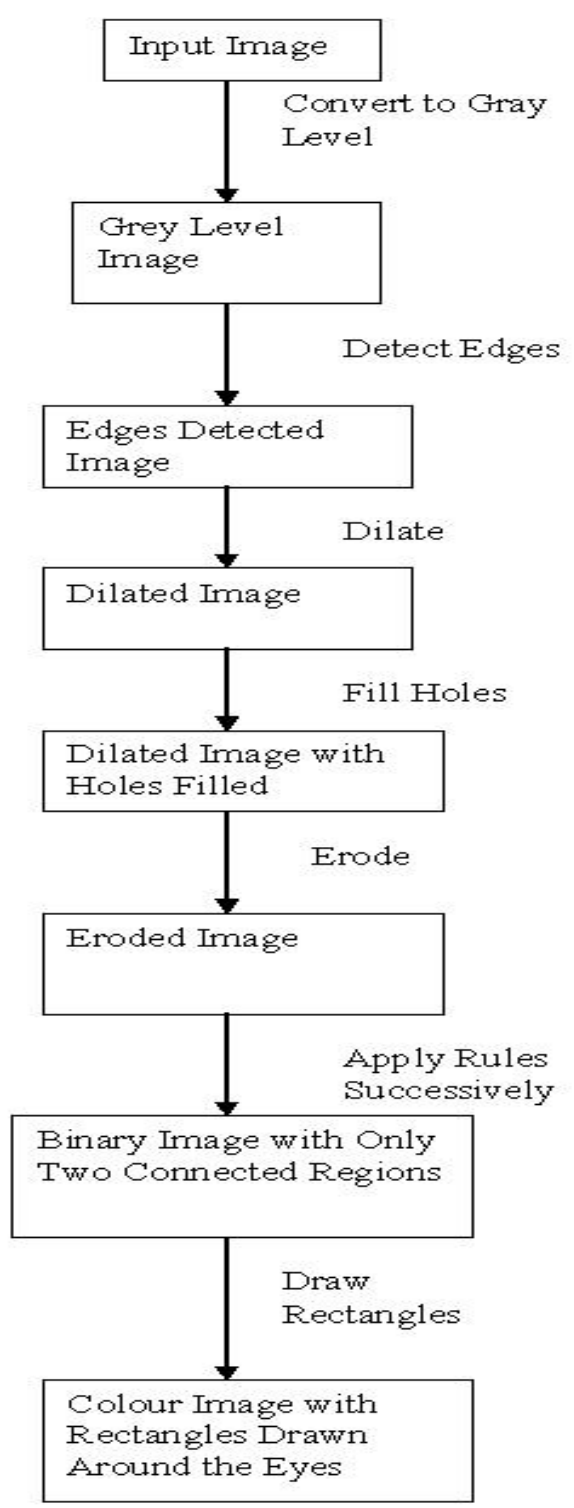

Figure 2 Flow Diagram of the Algorithm

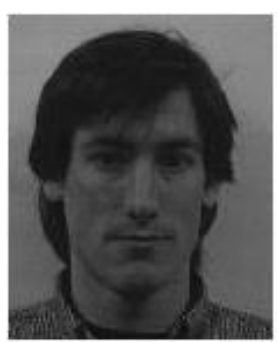

(a)

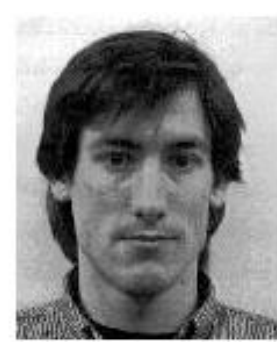

(b)
Figure 3 Lighting Compensation (a) Original Image (b) LightCompensated Image 


\section{B. Edge detection}

Sobel operator is applied to detect the edges. Since the edges in eye regions are sharp enough, so sobel edge detector is used. Figure 4 shows the result of edge detection.

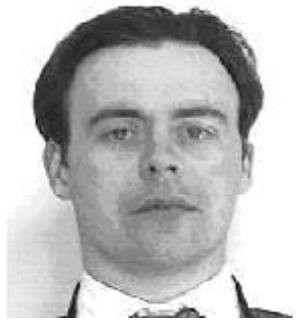

(a)

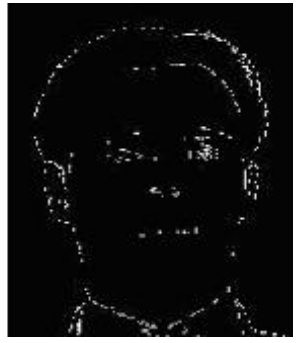

(b)
Figure 4 Edge Detection (a) Grey Level Image (b) Detected Edges

\section{Dilation, holes filling and Erosion}

Morphological dilation is used to enhance the eyes region edges. The image is dilated twice to get the holes in eyes filled. Disk shape structuring element with radius 3 has been used in our experiments. After this step, eyes become filled regions. However, sometime small holes are left inside the eye region which may harm the algorithm during erosion. For that the small holes inside eye regions need to be filled before erosion. To do this holes filling, the negative of dilated image is taken and the regions having very small area (there is a high probability that these regions are holes) are discarded. The negative of the image is taken again to get the image with small holes removed. During the dilation phase, some unwanted edges are also enhanced. To remove these unwanted edges, the image is eroded three times. Figures 5, 6 and 7 show dilation, erosion and hole filling respectively.

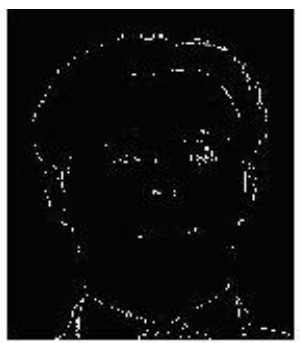

(a)

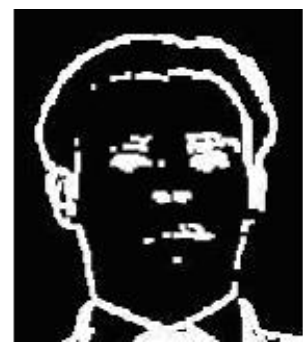

(b)
Figure 5 Morphological Dilation (a) Image Before Dilation (b) Image After Dilation

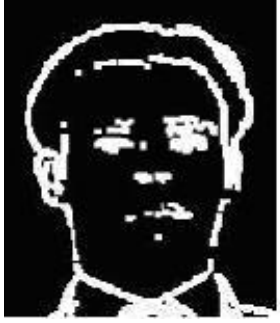

(a)

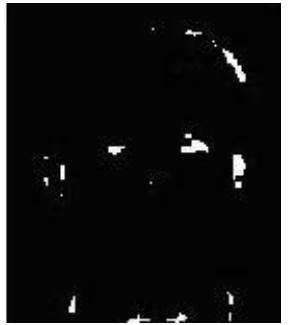

(b)
Figure 6 Morphological Erosion (a) Dilated Image (b) Eroded Image

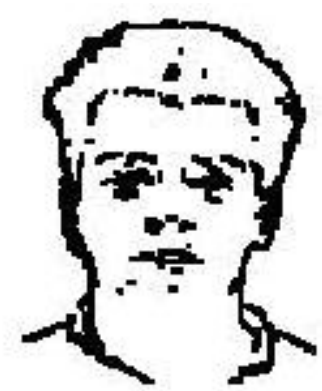

(c)

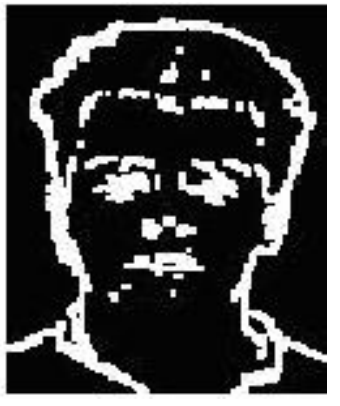

(d)

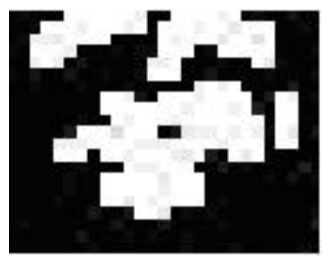

(e)

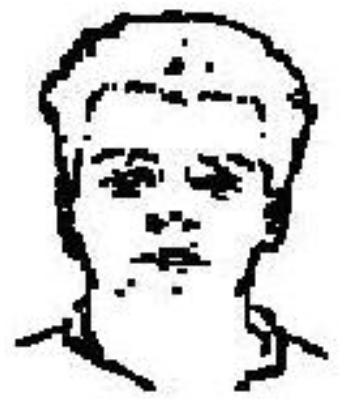

(b)

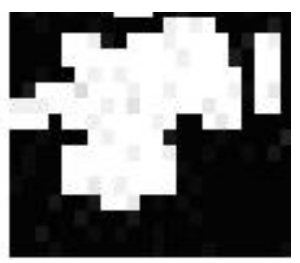

(f)

Figure 7 Holes Filling (a) Image With Small Holes (b) Negative Image With Holes (c) Negative Image With Holes Filled (d) Final Image With Holes Filled (e) Zoom Image of Right Eye Region With Holes (f) Zoom Image of Right Eye Region With Holes Filled 


\section{Eyes Extraction and Verification}

After these steps, the image contains several blobs. In order to find which of them are eyes, the following rules based on the shape and geometry of eyes are applied.

- The aspect ratios (width/height) of the eyes regions are between 0.8 and 4.0.

- The orientation angle of eyes is not greater than 45 degrees.

- Size of one eye shouldn't be greater than twice of the other eye and shouldn't be smaller than half of the other.

- The orientation of the two eyes shouldn't differ by more than 30 degrees.

- The line joining two eyes shouldn't have slope greater than 45 degree.

- The eyes are not too close to the borders of the image.

The above rules are applied successively. The region which doesn't conform to any of the rules is removed and thus is not considered for further rule tests. This successive testing scheme speeds up the process.

\section{IV.EXPERIMENTAL RESULTS}

The proposed idea was implemented in Matlab using image processing toolbox. The proposed algorithm was tested on PICS [2] facial images database. 80 photos of 50 individuals including both males and females of different ages and ethnicity were randomly selected. Most of them were of frontal faces. However, some faces were tilted to left or right. These are coloured images with Widths vary from 360 to 480 pixels while their heights vary from 480 to 540 pixels. On Intel core duo $1.60 \mathrm{GHz}$ Processor, the average processing time of our method was one second. Table 1 shows the results and percentage accuracy of our algorithm. The table shows that our algorithm is very accurate in initial blobs extraction stage. From the table it is also clear that accuracy is more when the input images are good illumination images.

In table 2, the proposed algorithm is compared with two other methods based on colour and illumination respectively $[1,5]$. Since these methods $[1,5]$ don't mention their eyes verification steps explicitly that's why we are comparing only the blob extraction not the final eye detector. Figure 8 shows some of the example images in which eyes were detected accurately. Figure 9 shows an example image in which our proposed method did better than the other two methods.

Table 1 Results of Our Proposed Method

\begin{tabular}{|l|l|l|l|l|l|}
\hline Step & Images Type & Total Images & $\begin{array}{l}\text { Correct } \\
\text { Detection }\end{array}$ & $\begin{array}{l}\text { Incorrect } \\
\text { detection }\end{array}$ & \% Accuracy \\
\hline Blobs Extraction & All Images & 80 & 76 & 4 & $95 \%$ \\
\cline { 2 - 6 } & $\begin{array}{l}\text { Good } \\
\text { Illumination } \\
\text { Images }\end{array}$ & 75 & 72 & 3 & $96 \%$ \\
\hline $\begin{array}{l}\text { Final Eyes } \\
\text { Extraction }\end{array}$ & All Images & 80 & 58 & 22 & $72.5 \%$ \\
\cline { 2 - 6 } & $\begin{array}{l}\text { Good } \\
\text { Illumination } \\
\text { Images }\end{array}$ & 75 & 58 & 17 & $77.33 \%$ \\
\hline
\end{tabular}

TABLE 2 COMPARISON WITH OTHER METHODS

\begin{tabular}{|l|l|l|l|l|l|}
\hline Method & Images Type & $\begin{array}{l}\text { Total } \\
\text { Images }\end{array}$ & $\begin{array}{l}\text { Correct } \\
\text { Detection }\end{array}$ & $\begin{array}{l}\text { Incorrect } \\
\text { Detection }\end{array}$ & $\begin{array}{l}\text { Percentage } \\
\text { Accuracy }\end{array}$ \\
\hline $\begin{array}{l}\text { Colour Based } \\
\text { Method [5] }\end{array}$ & All Images & 80 & 76 & 4 & $95 \%$ \\
\cline { 2 - 6 } & $\begin{array}{l}\text { Good } \\
\text { Illumination } \\
\text { Images }\end{array}$ & 75 & 72 & 3 & $96 \%$ \\
\hline $\begin{array}{l}\text { Illumination } \\
\text { Based Method [1] }\end{array}$ & All Images & 80 & 51 & 29 & $63.75 \%$ \\
\cline { 2 - 6 } & $\begin{array}{l}\text { Good } \\
\text { Illumination } \\
\text { Images }\end{array}$ & 75 & 56 & 19 & $74.66 \%$ \\
\hline $\begin{array}{l}\text { Edge Density } \\
\text { Based Method }\end{array}$ & All Images & 80 & 72 & 3 & $90 \%$ \\
\cline { 2 - 6 } & $\begin{array}{l}\text { Good } \\
\text { Illumination } \\
\text { Images }\end{array}$ & 75 & 72 & & \\
\hline
\end{tabular}




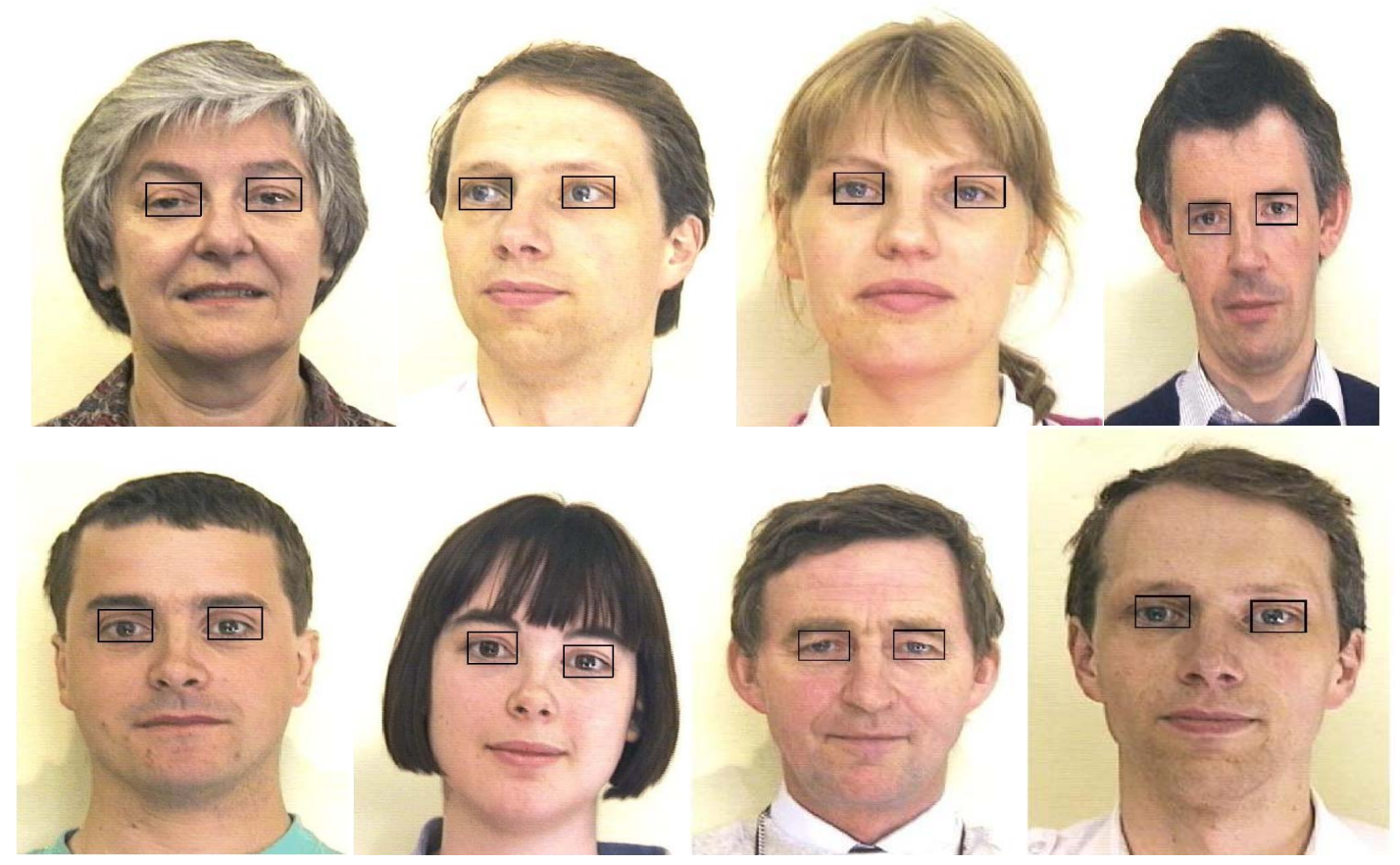

Figure 8 Examples of Images in which Eye were Correctly Detected

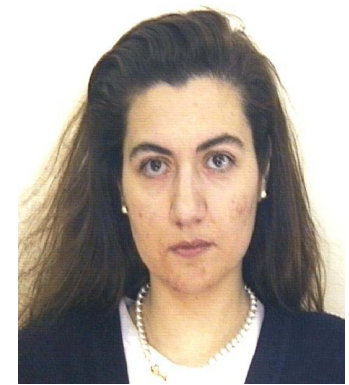

(a)

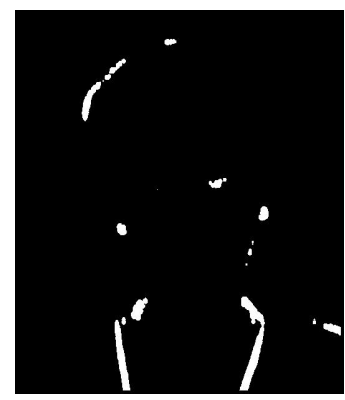

(c)

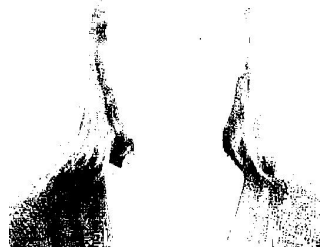

(b)

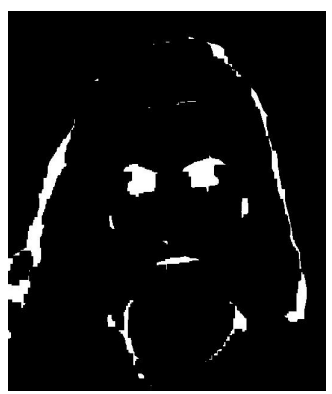

(d)

Figure 9 Example Image (a) Original Image (b) Blobs Detected by Method A i.e. Colour Based (c) Blobs Detected by Method B i.e. Illumination Based (d) Blobs Detected by Method C i.e. Our Method 


\section{CONCLUSIONS AND FUTURE WORK}

The paper proposes a novel method which is quite robust. The strength of the proposed method is its effectiveness for all ethnic people and its speed. Since the method doesn't rely on skin colour and works on colour variations which exist in all ethnic people. This makes the method applicable to all people. Except the first step i.e. conversion from colour to grey and edge detection, the rest of the computation is done in binary (two level) image which makes this method computationally very effective.

From the results, it is clear that our method doesn't do so well for badly illuminated images. So the plan is to improve the lighting compensation step to make the method robust even for badly illuminated images.

\section{REFERENCES}

[1] Hsu, R.L., Abdel-Mottaleb, M. \& Jain, A. 2002, "Face detection in color images", IEEE Transactions on Pattern Analysis and Machine Intelligence, vol. 24, no. 5, pp. 696-706.

[2] PICS, 2003. Psychological Image Collection at Stirling (PICS image data base). Available from <http://pics.psych.stir.ac.uk/>, University of Stirling Psychology Department.

[3] Morimoto, C., Koons, D., Amir, A. \& Flickner, M. 2000, "Pupil detection and tracking using multiple light sources", Image and Vision Computing, vol. 18, no. 4, pp. 331-335.

[4] Ebisawa, Y. \& Satoh, S. 1993, "Effectiveness of pupil area detection technique using two light sources and image difference method", Proceedings of the 15th Annual International Conference of the IEEE Engineering in Medicine and Biology Society, pp. 1268-1269.

[5] Chiang, C.C., Tai, W.K., Yang, M.T., Huang, Y.T. \& Huang, C.J. 2003, "A novel method for detecting lips, eyes and faces in real time", Real-Time Imaging, vol. 9, no. 4, pp. 277-287.

[6] Kawaguchi, T., Rizon, M. \& Hidaka, D. 2005, "Detection of eyes from human faces by Hough transform and separability filter", Electronics and Communications in Japan(Part II Electronics), vol. 88 , no. 5 , pp. 29-39.

[7] http://www.eye-floaters.com/theeye-anatomy.php 